\title{
Theorie der Modenlinienspektroskopie zur optischen Charakterisierung von Polymerschichten
}

\author{
Patrick Steglich*, Viachaslau Ksianzou, Claus Villringer, Silvio Pulwer und Sigurd Schrader
}

\section{Zusammenfassung}

Die Modenlinienspektroskopie kann zur hochpräzisen Charakterisierung von Polymerschichten bezüglich des Brechungsindex verwendet werden. Das vorliegende Manuskript behandelt die Theorie der Modenlinienspektroskopie und beginnt dabei mit den theoretischen Grundlagen eines planaren Wellenleiters, die zum Verständnis der Modenlinienspektroskopie notwendig sind. Die hier präsentierten expliziten Herleitungen können genutzt werden, um die Modenlinienspektroskopie so zu modifizieren, dass auch die Messung des Pockelseffektes möglich ist. Eine Anwendungsmöglichkeit soll in der zukünftigen Bestimmung des Pockelskoeffizienten bestehen, der für die Weiterentwicklung von polymerbasierten elektrooptischen Modulatoren benötigt wird. Zudem werden der Aufbau und die experimentelle Herangehensweisen beschrieben und die Brechungsindexbestimmung wird exemplarisch am Beispiel einer WirtGast-Polymerschicht demonstriert.

\begin{abstract}
M-Line spectroscopy can be used for high precision measurements of polymer layers. The present manuscript deals with the theory of m-line spectroscopy and thus begins with the theoretical background of a planar waveguide structure, which is necessary for a deeper understanding of $m$-line spectroscopy. The explicit derivations presented here can be used to modify the m-line spectroscopy in such a way that the measurement of the Pockels effect is also possible. One application is to be obtained in the future configuration of the Pockels coefficients, which is needed to optimize electro-optical modulators. In addition, the set-up and the experimental approach are described. Finally, a host-guest polymer layer is experimentally characterized in terms of the refractive index.
\end{abstract}

\section{Einführung}

Die Entwicklung photonischer Bauelemente in integrierten Schaltkreisen ist zu einem großen Teil auf die Möglichkeiten der Materialherstellung, der Probenstrukturierung und der Entwicklung sowie Kombination optischer Polymersysteme zurückzuführen, die neue Baukonzepte für ultraschnelle elektrooptische Modulatoren ermöglichen. Wellenleiter auf Polymerbasis eröffnen völlig neue Möglichkeiten für das Design und die Herstellung integrierter Optiken. So können beispielsweise ganze Schaltkreise über Verfahren wie Printed Electronics auf flexible Substrate gedruckt werden. Eine neue Entwicklung der letzten 15 Jahre ist die Silizium-Organik-Hybrid-Technologie (Gould et al. 2011; Alloatti et al. 2014; Korn et al. 2014). Diese Technologie nutzt CMOS-Herstellungsprozesse, um Siliziumwellenleiter zu strukturieren. Dieser Wellenleiter wird anschließend mit einem Polymer mit nichtlinearen optischen Eigenschaften (Steglich et al. 2015). Durch die Verbindung von Siliziumtechnologie und funktionellen Polymeren sind extrem energieeffiziente optische Schalter und Modulatoren herstellbar (Steglich et al. 2016).

Polymere mit einer hohen nichtlinearen optischen Suszeptibilität sind relativ kostengünstig herstellbar und leicht zu verarbeiten, was sie für industrielle Anwendungen interessant macht. Es ist daher notwendig, geeignete nichtlineare optische Polymermaterialien zu finden und zu charakterisieren. Dies impliziert auch, dass Vergleichskriterien und Messverfahren zur Charakterisierung solcher Polymerschichten entwickelt und evaluiert werden.
Im Rahmen des Projektes HOPBIT (Hybride Silizium-organische Photonik für die hochbitratige Datenübertragung) der TH Wildau sollen verschiedene Polymersysteme hinsichtlich ihrer optischen und elektrooptischen Eigenschaften untersucht werden. Dies beinhaltet die Untersuchung verschiedener Wirt-Gast-Systeme bestehend aus optischen Polymermatrizen und konventionellen sowie neuartigen optisch nichtlinearen Chromophoren. Die Modenlinienspektroskopie wurde dafür ausgewählt, weil sie den Brechungsindex bis auf die vierte Nachkommastelle genau bestimmen kann. In diesem Manuskript werden notwendige Grundlagen der Modenlinienspektroskopie vermittelt und ihre Vor- und Nachteile sowie mögliche Modifikationen bzw. Verbesserungen diskutiert. Am Beispiel eines Wirt-Gast 
Polymersystems, das aus dem Chromophor Dispersrot 1 und der Polymermatrix Polycarbonat besteht, wird der Brechungsindex bei verschiedenen Wellenlängen gemessen und daraus die Dispersion bestimmt.

\section{Theorie planarer Wellenleiter}

Zur Charakterisierung optischer Polymerschichten mittels Modenlinienspektroskopie ist es zweckmäßig, diese sie als planare Wellenleiter zu betrachten. Zum Verständnis der Messmethoden und der physikalischen Vorgänge während der Messungen wird in diesem Abschnitt die Theorie des planaren Wellenleiters beschrieben. Eine explizite Herleitung, wie sie in diesem Abschnitt ausgeführt ist, ist in keiner vonder den Autoren bekannten Literatur nicht zu finden. Einzelne theoretische Beschreibungen sind gibt es jedoch mit einem anderen Bezug in Standardwerken wie in Saleh \& Teich 2008 oder Hecht 2014 zu finden.

Die Führung einer elektromagnetischen Welle in einem dielektrischen Material mittels Totalreflexion ist das Grundprinzip eines planaren Wellenleiters. Aufgrund der Totalreflexion wird ein Lichtstrahl in dem Wellenleiter so geführt, dass keine Feldenergie an die umgebenden Medien abgegeben wird. Das heißt, es gibt keine Leistungsverluste an den Schichtoberflächen. Solch ein Wellenleiter besteht, wie in Abb. 1 zu sehen ist, im einfachsten Fall aus einer dielektrischen Schicht mit dem Brechungsindex $n_{f}$ und der Dicke $d$, welche die sich zwischen zwei Dielektrika mit den Brechungsindizes $n_{c}$ und $n_{\mathrm{s}}$ befindet.

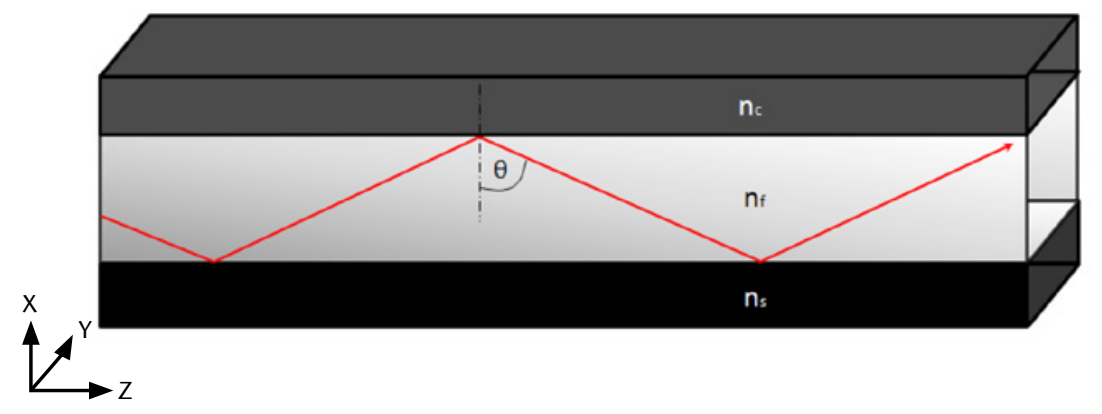

Abb. 1) Schematische Darstellung einer planaren Wellenleiterstruktur.

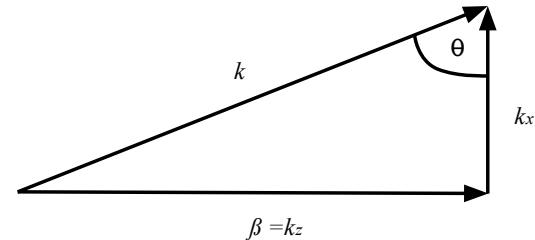

Abb. 2) Wellenvektorkomponenten kritische Winkel $\Theta_{\mathrm{g}}$ der Totalreflexion. Es folgt die Beziehung:

$\sin \Theta>\sin \Theta_{g}=\frac{n_{c, s}}{n_{f}}$

Daraus ergibt sich die Bedingung, dass die Tangentialkomponente der einfallenden Ausbreitungskonstante, welche die als Propagationskonstante $\beta$ bezeichnet wird, größer ist als die Ausbreitungskonstante einer freien Welle im angrenzenden Medium. Die Propagationskonstante gibt an, wie sich die Phase der Welle in eine bestimmte Raumrichtung ändert. $\beta$ gibt also die Phasenänderung der Welle in z-Richtung an. Damit lautet die Totalreflexionsbedingung:

$\beta=n_{f} k_{0} \sin \Theta>n_{c, s} k_{0}$

Im Folgenden wird ein Lichtstrahl, der sich in der $(x, z)$-Ebene unter dem Winkel $\Theta$ relativ zur $x$-Achse ausbreitet, durch seinen Wellenvektor $k$ beschrieben. $k_{0}$ ist die Ausbreitungskonstante und $\lambda$ die Wellenlänge des Lichtstrahls im Vakuum. Die Komponenten des Wellenvektors $k$ sind in Abb. 2 zu sehen und werden wie folgt beschrieben:

$$
\begin{aligned}
& k_{x}=k \cos \Theta \\
& k_{y}=0 \\
& k_{z}=k \sin \Theta
\end{aligned}
$$

Dabei gilt $\beta=k_{z}$ und $k=n_{f} k_{0}$. Eine ebene Welle breitet sich im Wellenleiter mit der Phasengeschwindigkeit $v_{p h}=\frac{c_{0}}{n_{f}}$ mit dem Winkel $\Theta$ aus, wobei $c_{0}$ die Vakuumlichtgeschwindigkeit ist. Grundsätzlich ist jeder Winkel möglich, solange die Totalreflexionsbedingung 2.2 eingehalten wird. Dadurch können, wie in Abb. 3 zu sehen ist, beliebig viele Strahlengänge entstehen. Jedoch interferieren die reflektierten Wellen nur bei bestimmten Reflexionswinkeln. Abhängig von Wellenleiterdicke und Brechungsindex entstehen stabile propagierende Wellen, die auch Moden genannt werden. Wellenleiter mit nur einem Strahlengang bzw. einer Mode werden als Monomoden-Wellenleiter bezeichnet. Bei mehreren Moden wird der Wellenleiter als Multimoden-Wellenleiter bezeichnet. Planare Wellenleiter sind Multimoden-Stufenindex-Wellenleiter. Bei dieser Art von Wellenleitern ändert sich der Brechungsindex sprungartig beim Übergang an den Grenzflächen zwischen dem hochbrechenden Kern (core) und dem niedrigbrechenden Mantel (cladding).

Moden höherer Ordnung, also Strahlengänge mit kleinem Reflexionswinkel $\Theta$, breiten sich langsamer aus als Moden niedrigerer Ordnung, da die Strahlen Wege unterschiedlicher Länge zurücklegen müssen. Abb. 3 zeigt diesen Effekt, der als Modendispersion

oder auch intermodale Dispersion bezeichnet wird.

\subsection{Eigenmoden planarer Wellenleiter}

Aufgrund der Wellennatur des Lichts können sich Moden nur in bestimmten Winkeln ausbilden. Wellen können miteinander interferieren und sich dabei verstärken (konstruktive Interferenz) oder abschwächen bzw. gegenseitig auslöschen (destruktive Interferenz). Eine Lichtwelle kann also nur bei konstruktiver Interferenz in einem 


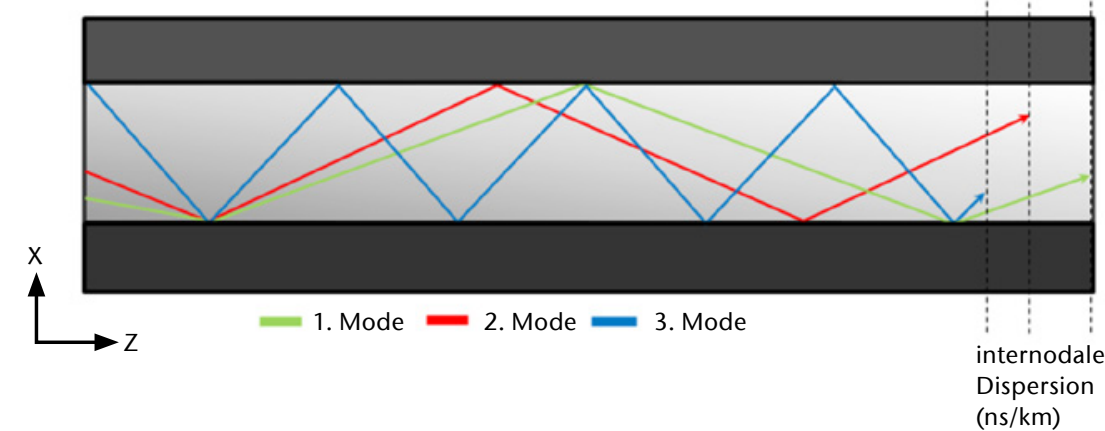

Abb. 3) Strahlengänge ausbreitungsfähiger Moden in einem planaren Wellenleiter.
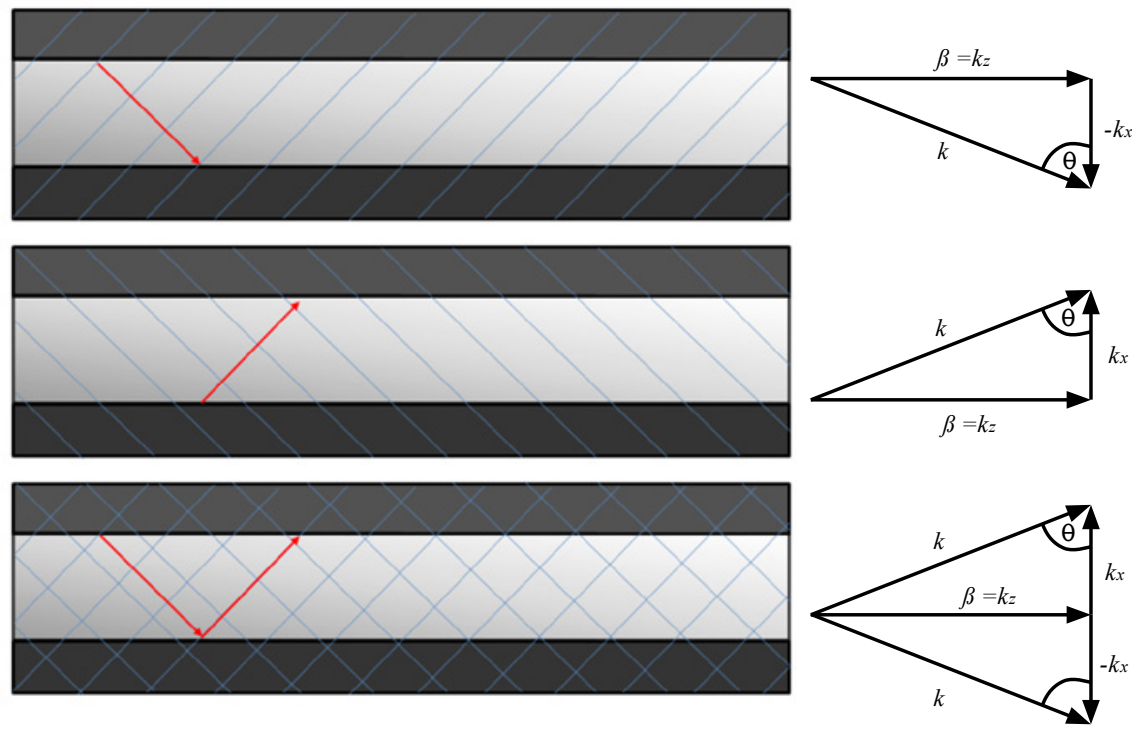

Abb. 4) Superposition zweier Felder in einem Wellenleiter, deren Wellenvektoren die gemeinsame Tangentialkomponente $\beta$ haben.

Wellenleiter geführt werden. Voraussetzung dafür ist die sogenannte Selbstkonsistenzbedingung.

Bei der Totalreflexion an einer dielektrischen Grenzfläche wird die Welle vom Einfallswinkel abhängig phasenverschoben. Diese Phasenverschiebung liegt im Bereich von $\varphi=0$ beim kritischen Winkel bis $\varphi=\pi$ bei streifendem Einfall. Grund dafür ist das evaneszente Feld.

Da die Welle durch Totalreflexion in dem Wellenleiter an zwei Grenzflächen hinund her reflektiert wird, kann das Feld in der Wellenleiterschicht als eine Superposition zweier Planwellen beschrieben werden, die durch Reflexion an den beiden Grenzflächen der Wellenleiterschicht ineinander übergehen. Jede dieser Planwellen besitzt, bei Vernachlässigung der Amplitude, die folgende Form:

$e^{-i\left[n_{f} k_{0}( \pm x \cos \Theta+z \sin \Theta)-\omega t\right]}=e^{-i\left(n_{f} k_{0} \pm x \cos \Theta\right)} e^{-i(\beta z-\omega t)}$.

Selbstkonsistenzbedingung ist eine definierende Eigenschaft der Eigenmoden eines Wellenleiters und legt die erlaubten Ausbreitungswinkel fest. Aufgrund der Superposition stellt die Welle in $\mathrm{x}$-Richtung eine stehende Welle dar. In z-Richtung breitet sie sich mit der Ausbreitungskonstante $\beta$ und der Phasengeschwindigkeit $v_{p h}$ aus. Im Folgenden wird exemplarisch mit einer TE-polarisierten Welle gearbeitet, und die Ergebnisse für TM-polarisierte Wellen werden nur angegeben, da die Herleitungen prinzipiell gleich sind.
Mit Gleichung 2.6 ist der Phasenterm einer im Film geführten (in y-Richtung homogenen) Welle gegeben. Damit folgt die Selbstkonsistenzbedingung für die phasenrichtige Überlagerung der $\mathrm{x}$ Komponenten der Teilwellen nach Hinund Rücklauf der Welle zwischen den beiden optischen Grenzflächen:

$2 k_{0} n_{f} d \cos \Theta-\phi_{T E}^{c}(\Theta)-\phi_{T E}^{s}(\Theta)=m \cdot 2 \pi$

$\phi_{T E}^{c}(\Theta)$ ist die Phasenverschiebung infolge der Reflexion einer TE-polarisierten Welle an der Grenzfläche zwischen zwei Dielektrika, in diesem Fall zwischen dem Film und dem Cladding, und ist abhängig vom Einfallswinkel $\Theta$ (Miller 1969). Gleiches gilt für die Phasenverschiebung zwischen Film und Substrat $\phi_{T E}^{c}(\Theta)$. Für den Fall eines symmetrischen Wellenleiters $\left(n_{c}=n_{s}\right)$ gilt für die Phasenverschiebung an den Grenzflächen: $\phi_{T E}^{c}(\Theta)+\phi_{T E}^{s}(\Theta)=$ $2 \phi^{c, s}{ }_{T E}(\Theta)$. Der erste Term $\left(2 k_{0} n_{f} d \cos (\Theta)\right.$ in Gleichung 2.7 ist, wie in Abb. $5 \mathrm{zu}$ sehen, die Phasenverschiebung zwischen den beiden Punkten A und B. Dies kann aus folgender Beziehung hergeleitet werden:

$\phi_{A B}-\phi_{A C}=\frac{4 \pi d n_{f}}{\lambda}=2 k_{0} n_{f} d \cos \Theta$

Die Phasenverschiebung von TM-Polarisierten polarisierten Wellen unterscheidet sich nur geringfügig. Um die Phasenverschiebung $\phi_{T E}^{c, s} z \mathrm{z}$ erhalten, wird zunächst der Amplitudenreflexionsfaktor $r_{T E}$ bzW. $r_{T M}$ betrachtet. Sie ergeben sich mit $\Theta$ als Einfallswinkel und $\alpha$ als Brechungswinkel aus den Maxwell'schen Gleichungen zu:

$r_{T E}=\frac{n_{f} \cos \theta-n_{c, s} \cos \alpha}{n_{f} \cos \theta+n_{c, s} \cos \alpha}=\frac{n_{f} \cos \theta-\sqrt{n_{t, s}^{2}-n_{f}^{2} \sin ^{2} \theta}}{n_{f} \cos \theta+\sqrt{n_{t, s}^{2}-n_{f}^{2} \sin ^{2} \theta}}$

$r_{T M}=\frac{n_{c, s} \cos \theta-n_{f} \cos \alpha}{n_{c, S} \cos \theta+n_{f} \cos \alpha}=\frac{n_{f} \cos \theta-n_{f} \sqrt{n_{C, s}^{2}-n_{f}^{2} \sin ^{2} \theta}}{n_{f} \cos \theta+n_{f} \sqrt{n_{C, S}^{2}-n_{f}^{2} \sin ^{2} \theta}}$

Für den Fall $\Theta \geq \Theta_{g}$ werden die Wurzeln imaginär und die reellen Amplitudenreflexionsfaktoren zu komplexen Zahlen. Damit ergibt sich der Amplitudenreflexionsfaktor einer TE-polarisierten Welle zu: 
$r_{T E}=\frac{n_{f} \cos \theta+i \sqrt{n_{f}^{2} \sin ^{2} \theta-n_{c, s}^{2}}}{n_{f} \cos \theta-i \sqrt{n_{f}^{2} \sin ^{2} \theta-n_{c, s}^{2}}}$

Die Abkürzungen

$$
\begin{array}{r}
\tilde{a}=n_{f} \cos \Theta \\
\tilde{b}=\sqrt{n_{f}^{2} \sin ^{2} \Theta-n_{c, s}^{2}}
\end{array}
$$

führen zur Definition des halben Phasensprungs:

$\tan \left(\frac{\phi_{T E}^{c, s}}{2}\right)=\frac{\tilde{b}}{\tilde{a}}$.

Daraus folgt, dass $\phi^{c, s}$ der jeweilige Phasensprung einer TE-polarisierten Welle an einem dielektrischen Medium ist. Für TM-polarisierte Wellen gilt analog dazu:

$\tan \left(\frac{\phi_{T M}^{c, s}}{2}\right)=\frac{n_{f}^{2}}{n_{c, s}^{2}} \frac{\tilde{b}}{\tilde{a}}$

Damit wird auch klar, dass die Amplitudenreflexionsfaktoren bei Totalreflexion den Betrag eins haben müssen, denn es gilt:

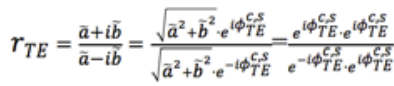

$$
\begin{aligned}
& =e^{i 2 \phi_{T E}^{c, s}}=\cos \left(2 \phi_{T E}^{c, s}\right)+i \sin \left(2 \phi_{T E}^{c, s}\right)
\end{aligned}
$$

Im Allgemeinen gibt es mehrere Lösungen für die Selbstkonsistenzbedingung 2.7, da in einem Multimoden-Wellenleiter mehrere Moden mit jeweils verschiedenen Modenwinkeln existieren. Jede dieser Moden besitzt einen diskreten Modenwinkel $\Theta_{M}$ und damit auch eine diskrete Ausbreitungskonstante:

$\beta_{m}=n_{f} k_{0} \sin \left(\Theta_{M}\right)$

sowie eine jeweilige Phasengeschwindigkeit:

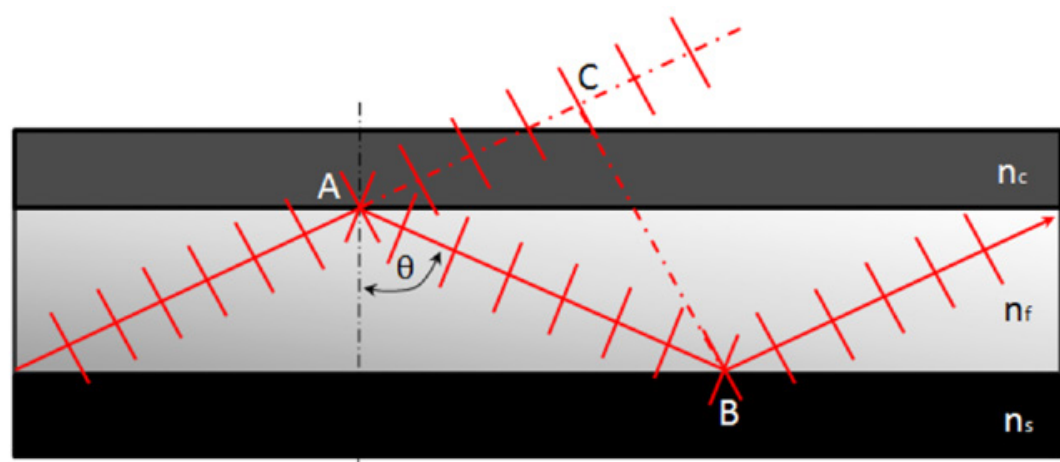

Abb. 5) Schematische Darstellung der Superposition zweier Felder in einem Wellenleiter. bei folgende Relationen:

$n_{c, s} \leq n_{e f f} \leq n_{f}$
Um Aussagen über die Ausbreitungsfähigkeit von Moden zu machen, ist es informativ, entweder die Ausbreitungskonstante $\beta(\omega)$ in Abhängigkeit von der Kreisfrequenz $\omega$ der Welle oder, davon abgeleitet, den sogenannten Phasenparameter $B$ als Funktion des Filmparameters $V$ aufzutragen.

Die Definition des effektiven Brechungsindex mag zwar anfänglich trivial erscheinen, doch seine Bedeutung ist sehr komplex. Auf den ersten Blick suggeriert das Wort "Brechungsindex“ eine Beziehung allein zum Material, obwohl der Begriff des effektiven Brechungsindex sowohl mit dem Material als auch mit der Mode verknüpft ist. Das heißt, für jede Mode als ausbreitungsfähige Welle existiert ein anderer effektiver Brechungsindex, obwohl das Material des Wellenleiterfilms jeweils dasselbe bleibt. Aus der Selbstkonsistenzbedingung 2.7 folgt, dass die Ausbreitungswinkel mit den Phasensprüngen an den Grenzflächen des Wellenleiterfilms verknüpft sind, diese aber ihrerseits von den Brechzahlen von Deckschicht und Substrat abhängen, wie die Gleichungen 2.14 und 2.15 verdeutlichen. Der effektive Brechungsindex ist also eine modenspezifische Größe, die von allen Brechzahlen der Wellenleiterstruktur abhängt. Die effektiven Brechungsindizes der einzelnen Moden können auch als Eigenwerte des Wellenleiters betrachtet werden. Bedingt durch Gleichung 2.1 gelten da-

Die Existenz eines effektiven Brechungsindex führt zur Realisierung von integriert-optischen Bauelementen, die den Lichtweg auch in der Ausbreitungsrichtung (Richtung der Ausbreitungskonstante $\beta_{m}$ ) beeinflussen.

Phasen- und Filmparameter sind folgendermaßen definiert (Reider 2013):

$B=\frac{n_{e f f}^{2}-n_{f}^{2}}{n_{f}^{2}-n_{s}^{2}}$

$V=k_{0} d \sqrt{n_{f}^{2}-n_{s}^{2}}$

Der Filmparameter $V$ kann wegen $V \propto k_{0}=\frac{2 \pi}{\lambda}=\frac{2 \pi}{c_{0}} v$ als normierte Frequenz oder Filmdicke und der Phasenparamter B entweder als normierte Ausbreitungskonstante oder normierter effektiver Brechungsindex bezeichnet werden. In beiden Definitionen fehlt noch die Abhängigkeit von der Deckschicht, also dem Cladding. Sie wird in einem weiteren Parameter, dem sogenannten Asymmetrieparameter $A_{T E}$ bzW. $A_{T M}$ für TE- und TM-Wellen, berücksichtigt:

$A_{T E}=\frac{n_{s}^{2}-n_{c}^{2}}{n_{f}^{2}-n_{s}^{2}}$

$A_{T M}=\frac{n_{f}^{4}}{n_{c}^{4}} \frac{n_{s}^{2}-n_{c}^{2}}{n_{f}^{2}-n_{s}^{2}}$

Mit diesen Parametern lässt sich die Selbstkonsistenzbedingung 2.7 in

$V \sqrt{1-B}=m \cdot \pi+\tan ^{-1} \sqrt{\frac{B}{1-B}}+\tan ^{-1} \sqrt{\frac{B+A}{1-B}}$

für TE- oder TM-Wellen umformen. Eine graphische Darstellung von $B$ als Funktion von $V$ wird als normiertes Dispersionsdiagramm bezeichnet. Jede Kurvenschar steht dabei für eine ausbreitungsfähige Mode und die Kurven innerhalb einer Schar entsprechen verschiedenen Asymmetrieparametern. Existieren für eine definierte Filmdicke $d$, die auf der Abszisse zu einem bestimmten Wert des Filmparameters führt, mehrere Moden, so haben die Moden mit kleinerem $m$ die größeren effektiven Brechungsindizes. Außerdem ist noch festzuhalten, dass alle Moden mit $m>0$ erst oberhalb einer bestimmten Grenzfrequenz ausbreitungsfähig sind. Anhand der 
Selbstkonsistenzbedingung 2.25 und mit Hilfe der definierten Parameter, können folgende Möglichkeiten abgeleitet werden, den effektiven Brechungsindex einer bestimmten Mode in einer Filmwellenleiterstruktur zu vergrößern:

\section{- Erhöhung der Filmdicke $d$,}

- Erhöhung der Materialbrechzahl der Deckschicht und des Substrats, $n_{c}$ und $n_{s}$, zur Verringerung der Asymmetrie,

- Erhöhung der Materialbrechzahl des Wellenleiterfilms $n_{f}$.

Diese Abhängigkeiten kann können zum Beispiel bei der Herstellung von Filmlinsen und Streifenwellenleitern zu Nutze gemachtgenutzt werden. Die normierte Grenzfrequenz $V_{m}$ der Moden ergibt sich aus Gleichung 2.25 für $B=0$. Für $m=0$ erhält man die normierte Grenzfrequenz der Grundmode:

$V_{0}=\tan ^{-1} \sqrt{A_{T E}}$

Daraus folgt, dass die normierte Grenzfrequenz der Grundmode nur bei symmetrischen Wellenleitern null beträgt. Die normierte Grenzfrequenz höherer Moden ist gegeben durch:

$V_{m}=V_{0}+m \cdot \pi$

Damit ergibt sich für $V_{0} \ll V_{m}$ ein genäherter Ausdruck für die Anzahl der ausbreitungsfähigen TE-Wellen mit:

$M=\frac{v_{m}}{\pi}=\frac{2 d}{\lambda} \sqrt{n_{f}^{2}-n_{s}^{2}}$

Die Selbstkonsistenzbedingung 2.7 kann für den Fall eines symmetrischen Wellenleiters auch noch in eine etwas kompaktere Form umgeschrieben werden. Dazu werden üblicherweise zwei weitere Parameter definiert (Reider 2013):

$$
\begin{aligned}
u & =d k_{0} \sqrt{n_{f}^{2}-n_{e f f}^{2}} \\
& =d k_{0} n_{f} \cos \theta=d \sqrt{k_{0}^{2} n_{f}^{2}-\beta^{2}} \\
w & =d k_{0} \sqrt{n_{e f f}^{2}-n_{s}^{2}}=d \sqrt{\beta^{2}-k_{0}^{2} n_{f}^{2}}
\end{aligned}
$$

so dass $u^{2}+w^{2}=V^{2}$ gilt. Mit diesen normierten Werten lautet Gleichung 2.14:

$\tan \left(\phi_{r}\right)=\frac{w}{u}$

Die Selbstkonsistenzbedingung 2.7 lautet nun:

$\tan (u-m \cdot \pi)=\frac{w}{u}$

bzw. mit $u^{2}+w^{2}=V^{2}$ :

$\tan (u-m \cdot \pi)=\frac{\sqrt{V^{2}-u^{2}}}{u}$

In dieser Gleichung ist bei gegebenem Wellenleiter und gewählter Wellenlänge die normierte Frequenz $V$ konstant und die beiden Parameter $u$ und $w$ hängen nur von $\beta$ und damit nach Gleichung 2.17 von dem diskreten Modenwinkel $\sin \Theta_{m}$ ab. Werden alle Parameter eingesetzt und wird die Beziehung für den Grenzwinkel der Totalreflexion

$\sin \Theta_{g}=\frac{\sqrt{n_{f}^{2}-n_{s}^{2}}}{u}$

genutzt, so erhält man folgenden Ausdruck für die Selbstkonsistenzbedingung eines symmetrischen Wellenleiters (Reider 2013):

$\left(d n_{f} k_{0} \sin \Theta-m \cdot \frac{\pi}{2}\right)=\sqrt{\frac{\sin ^{2} \theta_{g}}{\sin ^{2} \Theta}-1}(2.35)$

\section{Theoretische Grundlagen der Modenlinienspektroskopie}

Planare Wellenleiter können durch ihren Satz ausbreitungsfähiger Moden hinsichtlich ihrer Schichtdicke und ihres Filmbrechungsindex charakterisiert werden (Monneret et al. 2000). Jede Mode ist durch ihren effektiven Brechungsindex gekennzeichnet, der für Wellenleiter mit homogenem Brechungsindexverlauf durch den reellen Filmbrechungsindex und den Ausbreitungswinkel der Mode gegeben ist:

$n_{\text {eff }, m}=n_{f} \sin \left(\Theta_{M}\right)$

Die effektiven Brechungsindizes der geführten Moden lassen sich über das in der integrierten Optik häufig genutzte Verfahren der Modenlinienspektroskopie ermitteln, das auf der selektiven Anregung einzelner Wellenleitermoden mit Hilfe eines hochbrechenden Prismas basiert. Eine Prin- zipdarstellung der Prismenkopplung ist in Abb. 6 gezeigt. Ein Laserstrahl trifft unter einenin einem bestimmten Winkel auf das hochbrechende Prisma und wird dabei über das Prisma in den Wellenleiterfilm eingekoppelt. Aufgrund der Konsistenzbedingung nach Gleichung 2.7 kann der Laserstrahl allerdings nur bei bestimmten Winkeln, den sogenannten Kopplungswinkeln $\Theta_{M}$, in den Wellenleiter eingekoppelt werden.

Um diese diskreten Winkel $\Theta_{M}$ zu ermitteln, wird der Winkel $\alpha$ so lange variiert, bis eine ausbreitungsfähige Mode angeregt wird. Der Kopplungswinkel ist jener Winkel, bei dem die nicht in die Schicht eingekoppelte Intensität ein Minimum besitzt. In Abb. 7 ist ein Beispiel für den Intensitätsverlauf einer Polycarbonatschicht zu sehen.

Der effektive Brechungsindex der angeregten Mode ist abhängig von dem gemessenen Winkel $\alpha_{m}$. Durch das Snellius'sche Brechungsgesetz und mit Hilfe von trigonometrischen Beziehungen kann Gleichung 3.1 so umgeschrieben werden, dass der effektive Brechungsindex nur noch von dem Brechungsindex des Prismas $n_{p}$, dem Prismawinkel $\vartheta$ und dem gemessenen Winkel $\alpha$ abhängt (Ulrich \& Torge 1973):

$$
\begin{aligned}
n_{\text {eff }, m} & =n_{p}\left[\frac{\pi}{2}-\frac{\vartheta}{2}+\sin ^{-1}\left(\frac{\sin \alpha_{m}}{n_{p}}\right)\right] \\
& =n_{p}\left[\varepsilon+\sin ^{-1}\left(\frac{\sin \alpha_{m}}{n_{p}}\right)\right]
\end{aligned}
$$

Mit Gleichung 3.2 und Gleichung 2.25 erhält man ein Gleichungssystem, welches das iterativ zu lösen ist. Dadurch lässt lassen sich der Filmbrechungsindex $n_{f}$ und die Schichtdicke $d$ des untersuchten Wellenleiters berechnen. Es ist allerdings dabei allerdings zu beachten, dass mindestens zwei Moden notwendig sind, da das Gleichungssystem sonst unterbestimmt ist. Die für das jeweilige Polymer typischen Modellparameter $V, B$ und $A$ sind nur von dem effektiven Brechungsindex $n e_{f f}$ abhängig. Die Modelparameter werden also aus den experimentellen Daten extrahiert und durch das Gleichungssystem numerisch angepasst. Zum Lösen des Gleichungssystems sind allerdings Startwerte für $d$ und $n e_{f f}$ nötig. Als Näherungswert für die Filmschichtdicke kann die Probe vorher mit einem geeigneten Messmittel, wie z. B. 
mit einem Profilometer, vermessen werden. Indem Gleichung Gleichung 2.28 umgestellt wird, ist es mit der gemessenen Schichtdicke möglich, eine gute Näherung für den Filmbrechungsindex zu erhalten (Ulrich \& Torge 1973):

$n_{f}=\sqrt{\left(\frac{M \lambda}{2 d}\right)^{2}+n_{s}^{2}}$.

\section{Experimenteller Aufbau der Moden- linienspektroskopie}

Der experimentelle Aufbau der Modenlinienspektroskopie ist in Abb. 8 schematisch dargestellt. Der Laserstrahl wird durch einen Chopper pe-

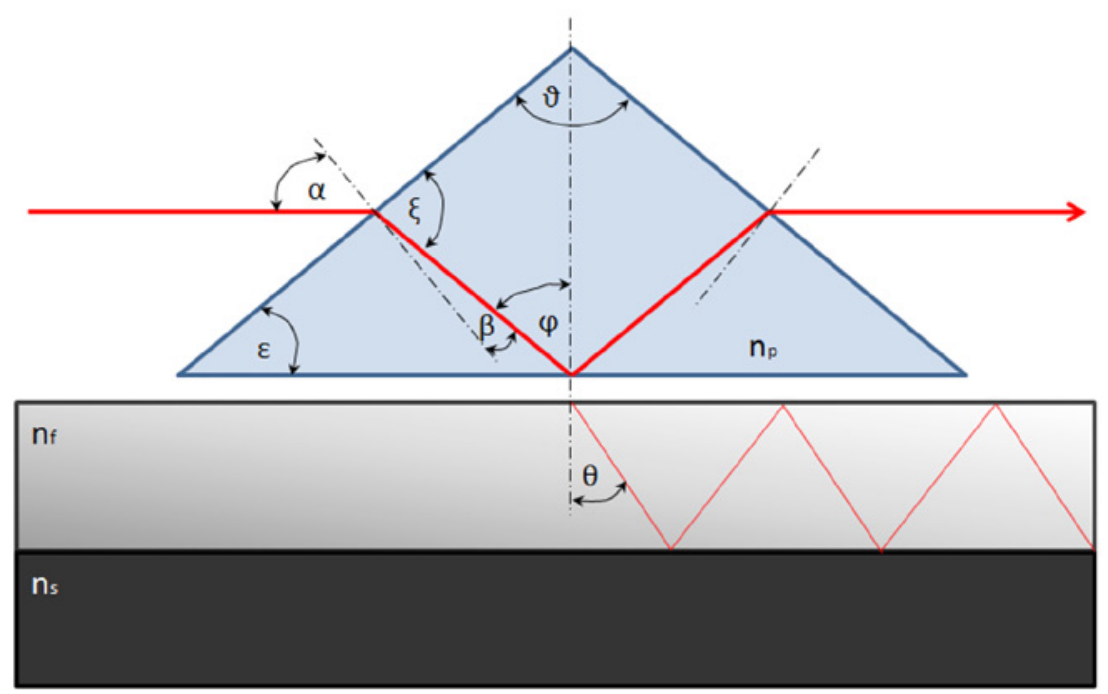

Abb. 6) Prinzip der Prismenkopplung: Über ein hochbrechendes Prisma wird ein Laserstrahl bei einem bestimmten Winkel (Kopplungswinkel) in den Wellenleiter eingekoppelt.

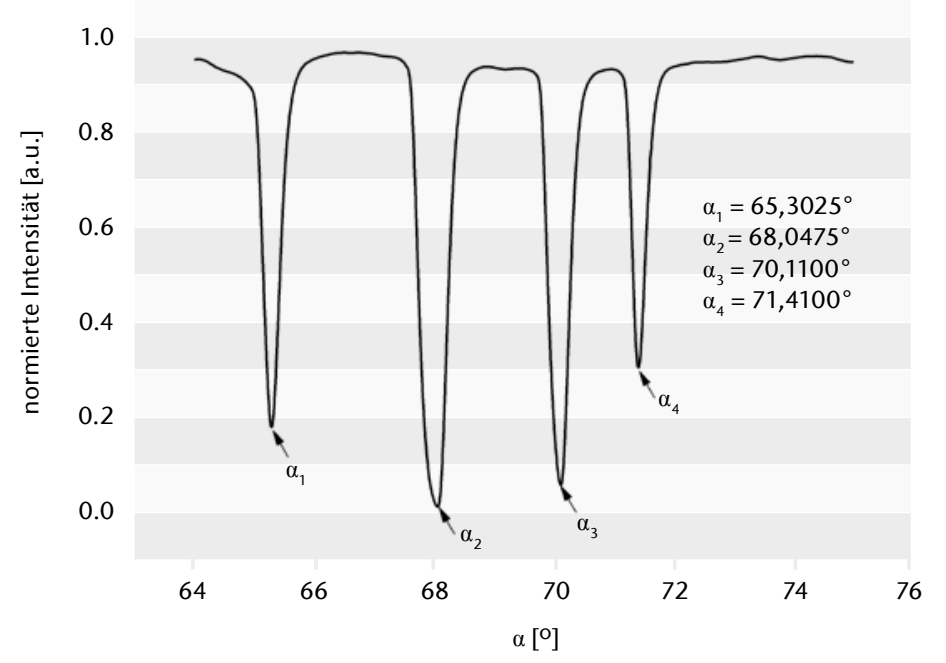

Abb. 7) Charakteristischer Intensitätsverlauf der Modenlinienspektroskopie. Bei dem Winkel, bei dem die Intensität abfällt, kann sich durch konstruktive Interferenz eine Mode ausbilden. Der Wellenleiter besteht aus einer Polycarbonatschicht auf einem Glassubstrat (BK7) und wurde mit einem Nd:YAG Laser vermessen. mit der Brechungsindex und die Schichtdicke polarisationsabhängig berechnet werden können.

3. Blende: Die Blende dient der Begrenzung des Laserstrahldurchmessers, um Inhomogenitäten am Rand des Strahlprofils zu unterbinden.

4. Linsen: Mit Hilfe von Linsen wird der Laserstrahl auf das Prisma bzw. auf den Photodetektor fokussiert.

5. Prisma: Das Prisma dient zur Einkopplung des Laserstrahls in den Filmwellenleiter. Das verwendete Prisma sollte möglichst hochbrechend sein. Ein symmetrisches Prisma eignet sich gut für aufgedampfte oder aufgesputterte Filme mit leichten Inhomogenitäten, wobei das Substrat dünn und flexibel sein kann.

6. Drehtisch: Der Drehtisch ist mit einem Goniometer verbunden, womit die exakte Lage des Prismas zum Laserstrahl bestimmt werden kann. Er besitzt separate Positioniereinheiten, mit dem denen das Prisma und der Detektor einzeln oder gemeinsam angesteuert werden können.

7. Detektor: Mittels einer Photodiode wird die Intensität des reflektierten Laserstrahls in Abhängigkeit des von Winkels $\alpha$ aufgenommen.

8. Lock-In in-Amplifier: Der Lock-In in-Amplifier dient zur Verstärkung des Signals an der Photodiode und ist mit einem Computer verbunden, der diese Signale auswertet.

\section{Experimenteller Ablauf der Moden- linienspektroskopie}

Der experimentelle Ablauf der Modenlinienspektroskopie kann in folgende Punkte zusammengefasst werden:

1. Zunächst wird die Intensität des Laserstrahls gemessen. Dabei fährt der Drehtisch den gewünschten Winkelbereich ab und der Photodetektor nimmt die Intensität des Laserstrahls auf. 
2. Als nächstes Nächstes müssen die Winkel $\alpha_{m}$ bestimmt werden, bei denen die Intensität ein Minimum besitzt (siehe Abb. 7).

3. Mit diesen Winkeln werden die effektiven Brechungsindizes aus Gleichung 3.2 berechnet.

4. Außerdem ist noch ein Startwert für die Schichtdicke nötig. Diese muss mit einem geeigneten Verfahren wie $z$. B. einem Profilometer oder einem Atomkraftmikroskop ermittelt werden.

5. Handelt es sich um eine Probe mit unbekanntem Brechungsindex, kann mit Hilfe der Schichtdicke ein Startwert für den Filmbrechungsindex berechnet werden. Wenn der Brechungsindex allerdings bekannt ist, kann diese Gleichung auch für die Schichtdicke umgestellt werden, so dass diese nicht extra ermittelt werden muss.

6. Jetzt ist es möglich, dass Gleichungssystem iterativ aus den experimentell ermittelten Messwerten zu lösen und damit einen exakten Wert für die Schichtdicke und den Filmbrechungsindex zu erhalten.

7. Da es Messwertabweichungen aufgrund von Inhomogenitäten im Material an den einzelnen Messpunkten geben kann, sollten mehrere Punkte auf der Probe gemessen und anschließend statistisch mit einer geeigneten Software ausgewertet werden.

\section{Experimentelle Ergebnisse}

In diesem Abschnitt soll exemplarisch eine Messung des Brechungsindex bei verschiedenen Wellenlängen demonstriert werden. Brechungsindex und Absorption sind wellenlängenabhängig, was in der Optik als Dispersion bezeichnet wird. Das Dispersionsverhalten von optischen Polymeren kann mit der Modenlinienspektroskopie durch den Einsatz unterschiedlicher Laser gemessen werden. Für den Spektralbereich von 400 bis $1.600 \mathrm{~nm}$ ist die Cauchy-Näherung eine sehr gute Näherung für das Dispersionsverhalten

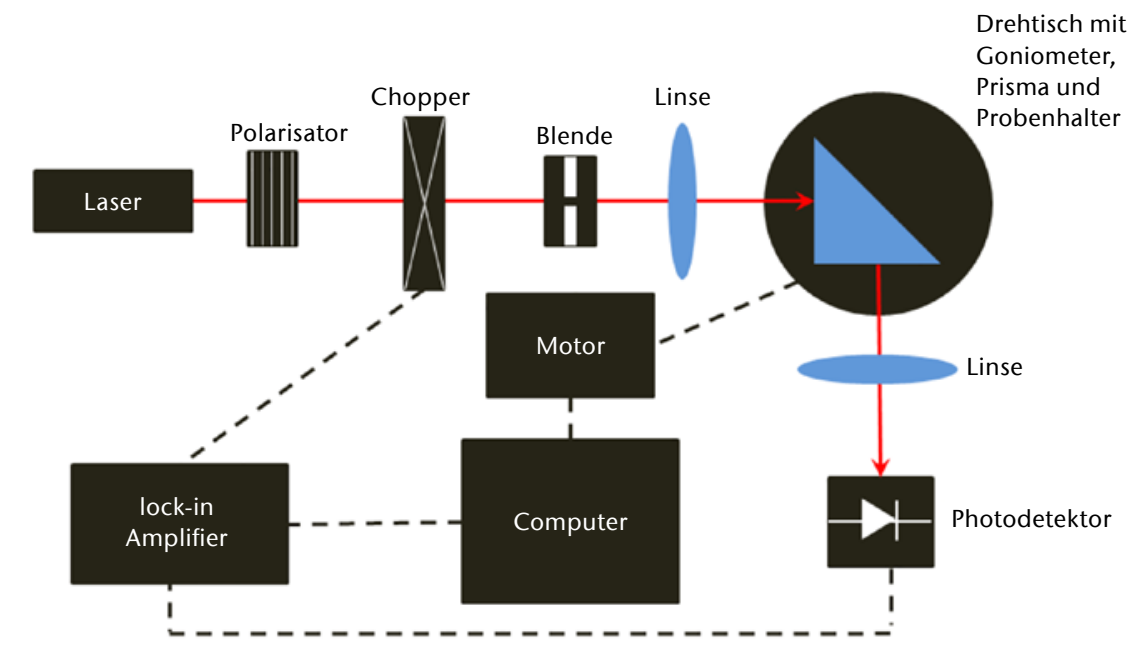

Abb. 8) Schematischer Aufbau der Modenlinienspektroskopie. Der Laserstrahl wird polarisiert und auf das Prisma fokussiert, auf dem sich die Probe befindet. Nach der Reflexion am Prisma wird die nicht in die Schicht eingekoppelte Intensität des Laserstrahls über einen Photodetektor gemessen.

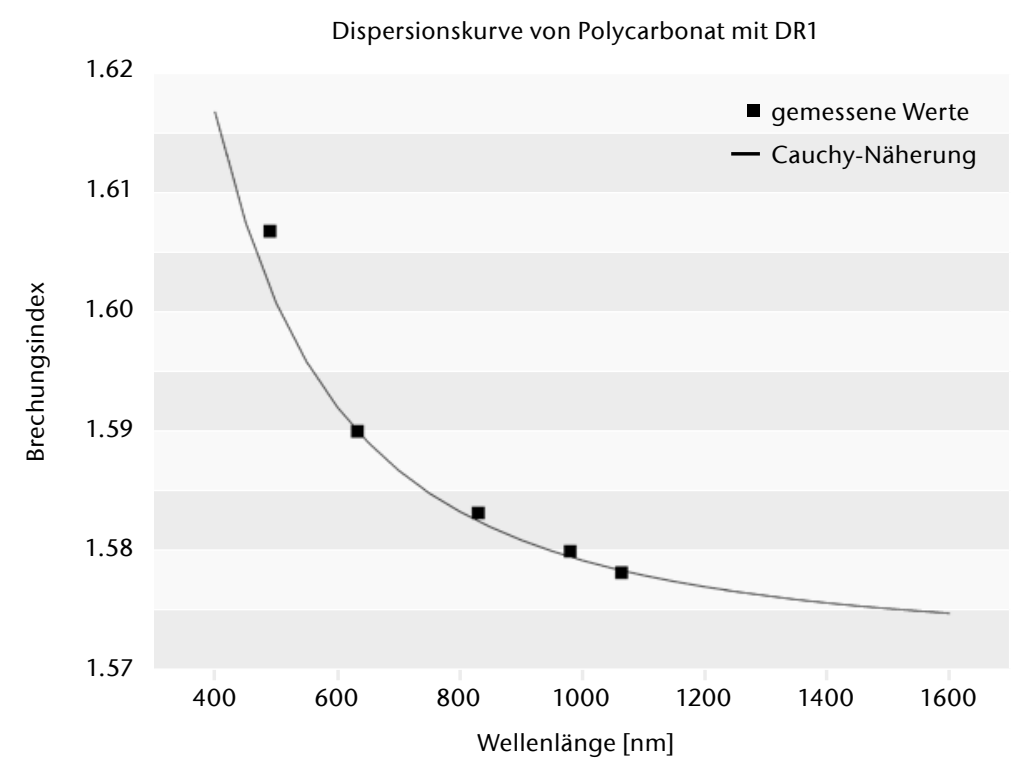

Abb. 9) Brechungsindizes von Polycarbonat mit DR1 (5 Massenprozent) bei verschiedenen Wellenlängen und eine durch die Cauchy-Näherung angepasste Dispersionskurve.

und wird auch im Folgenden verwendet. Die hier präsentierten Messergebnisse sind nicht auf ihre Reproduzierbarkeit geprüft und dienen lediglich zur experimentellen Veranschaulichung der Modenlinienspektroskopie. Zur Berechnung des Brechungsindex und der Schichtdicke ist es bei der Modenlinienspektroskopie nötig, die transzendente Gleichung der Selbstkonsistenzbedingung $2.25 \mathrm{zu}$ lösen. Dazu ist allerdings ein Startwert für die Schichtdicke Voraussetzung. Dieser wurde mit einem DEKTAK DEKTAK-150 150-(Veeco-)Profilometer ermittelt. Das Profilometer arbeitet taktil mit einer Diamantspitze, welche die einen
Radius von 12,5 $\mu \mathrm{m}$ besitzt. Die Probe wird elektromechanisch unter der Diamantspitze bewegt, um so ein Höhenprofil zu erstellen. Die gemessene Schichtdicke beträgt 3,552 $\mu \mathrm{m}$.

Abb. 9 zeigt den gemessenen Brechungsindex von Polycarbonat mit Dispersrot Dispersrot 1 (5 Massenprozent) bei verschiedenen Wellenlängen und eine durch die Cauchy-Näherung angepasste Dispersionskurve. Die größte Fehlerquelle liegt darin, dass die Probe an das Prisma gedrückt werden muss, so dass eine Einkopplung des Lichts in die Polymerschicht möglich ist. Dadurch kann sich jedoch die Schichtdicke leicht verringern, was zu 
einem systematischen Fehler führt. Frühere Arbeiten haben eine theoretische Genauigkeit des Messverfahrens von $\Delta \alpha=10^{-5}$ demonstriert (Monneret et al. 2000). Der Einfluss des Anpressdrucks auf die absolute Genauigkeit des Messverfahrens muss noch in weiteren Studien geprüft werden.

\section{Zusammenfassung und Ausblick}

Die theoretischen Grundlagen der Modenlinienspektroskopie wurden in diesem Skript beschrieben. Durch die explizite Betrachtung planarer Wellenleiter konnten die wesentlichen Formeln und Gleichungen für TE- und TM-polarisiertes Licht hergeleitet werden. Die daraus abgeleitete Selbstkonsistenzbedingung in einem planaren Wellenleiter wurde genutzt, um die Schichtdicke und den Brechungsindex zu berechnen. Dazu wurden mit der Modenlinienspektroskopie bei verschiedenen Wellenlängen die Intensitätsverteilungen in Abhängigkeit vom Einfallswinkel gemessen. Eine explizite Anleitung zur experimentellen Ermittlung der Intensitätsverteilungen sowie eine Näherungsformel zur Berechnung der minimalen Schichtdicken wurden beschrieben und theoretisch hergeleitet. Durch die Betrachtung der einzelnen Polarisationszustände (TE und TM) kann auch die optische Anisotropie mit Hilfe der Modenlinienspektroskopie bestimmt werden. In dieser Arbeit wurde exemplarisch anhand einer Polymerschicht die Ermittlung des Brechungsindex demonstriert. Die Schichtdicke wurde dabei mit einem Profilometer gemessen. Unsere Ergebnisse zeigen zwar die hochpräzise Messung des Brechungsindex, müssen jedoch auf ihre Reproduzierbarkeit in zukünftigen Arbeiten geprüft werden.

Weitere Forschungsarbeiten an der TH Wildau werden sich mit der theoretischen und experimentellen Bestimmung des Pockelseffektes von nichtlinear optischen Polymeren mittels der Modenlinienspektroskopie beschäftigen. Dieser Effekt ist für eine effektive Weiterentwicklung von elektrooptischen Modulatoren, insbesondere für die Schaltgeschwindigkeit und den Spannungsbedarf, von wesentlicher Bedeutung. Dazu können die hier präsentierten theoretischen Grundlagen genutzt werden, um die Brechungsindexänderung in Abhängigkeit von einem elektrischen Feld zu bestimmen. So kann der Pockelseffekt, also die Änderung des Brechungsindex durch Anlegen eines elektrischen Feldes, mit Gleichung 3.2 bestimmt werden, wenn der Brechungsindex vor und nach dem Anlegen des elektrischen Feldes gemessen wird. Ein besonderer Vorteil ist, dass auch die Dispersion, also die Wellenlängenabhängigkeit, des Pockelseffektes analog zu dem Beispiel in Abb. 9 gemessen werden kann. Des Weiteren soll in Zukunft auch die Temperaturabhängigkeit des Brechungsindex durch die Modenlinienspektroskopie bestimmt werden. Dafür wird der Probenhalter mit einem Heizelement modifiziert.

\section{LITERATUR}

Alloatti L, Palmer R, Diebold S, Pahl KP, Chen B, Dinu R, Fournier M, Fedeli J-M, Zwick T, Freude W, Koos C, Leuthold I (2014) $100 \mathrm{GHz}$ silicon-organic hybrid modulator. Light Sci Appl 3(5):e173. doi: 10.1038/Isa.2014.54

Gould M, Baehr-Jones T, Ding R, Huang S, Luo J, Jen AK-Y, Fedeli I-M, Fournier M, Hochberg M (2011) Silicon-polymer hybrid slot waveguide ring-resonator modulator. Opt Express 19(5):3952-3961. doi: 10.1364/ OE.19.003952

Hecht E (2014) Optik. De Gruyter Studium. De Gruyter, Berlin. ISBN: 978-3110526646

Korn D, Jazbinsek M, Palmer R, Baier M, Alloatti L, Yu H, Bogaerts W, Lepage G, Verheyen P, Absil P, Guenter P, Koos C, Freude W, Leuthold I (2014) Electro-Optic Organic Crystal Silicon High-Speed Modulator. IEEE Photonics J 6(2):1-9. doi: 10.1109/JPHOT.2014.2314113

Miller SE (1969) Integrated Optics. An Introduction. Bell System Technical Journal 48(7):2059-2069. doi: 10.1002/j.1538-7305.1969.tb01165.x

Monneret S, Huguet-Chantôme P, Flory F (2000) m-lines technique Prism coupling measurement and discussion of accuracy for homogeneous waveguides. J. Opt. A: Pure Appl. Opt. 2(3):188-195. doi: 10.1088/1464$4258 / 2 / 3 / 304$

Reider GA (2013) Photonik. Eine Einführung in die Grundlagen, 3. Aufl. Springer, Wien. ISBN: 9783709115206

Saleh BEA, Teich MC (2008) Grundlagen der Photonik, 2., vollst. überarb. und erw. Aufl. Lehrbuch Physik. Wiley-VCH, Weinheim. ISBN: 978-3527406777

Steglich P, Mai C, Stolarek D, Lischke S, Kupijai S, Villringer C, Pulwer S, Heinrich F, Bauer J, Meister S, Knoll D, Casalboni M, Schrader S (2015) Novel Ring Resonator Combining Strong Field Confinement With High Optical Quality Factor. IEEE Photon Technol Lett 27(20):2197-2200. doi: 10.1109/LPT.2015.2456133

Steglich P, Mai C, Stolarek D, Lischke S, Kupijai S, Villringer C, Pulwer S, Heinrich F, Bauer J, Meister S, Knoll D, Casalboni M, Schrader S (2016) Partially slotted silicon ring resonator covered with electro-optical polymer. In: Vivien L, Pavesi L, Pelli S (eds), Sunday 3 April 2016, Brussels, Belgium. SPIE Proceedings. SPIE, 98910R. doi: $10.1117 / 12.2217725$

Ulrich R, Torge R (1973) Measurement of thin film parameters with a prism coupler. Appl Opt 12(12):29012908. doi: 10.1364/AO.12.002901

\section{AUTOREN}

Dr. Patrick Steglich*

Dr. Viachaslau Ksianzou

Claus Villringer, M.Eng.**

Silvio Pulwer, M.Eng.**

Prof. Dr. Sigurd Schrader

Forschungsgruppe für Photonik, Laser \&

Plasmatechnologien

Technische Hochschule Wildau

* Gastwissenschaftler am Leibniz-Institut

für innovative Mikroelektronik IHP

** University of Rome „Tor Vergata“

E-Mail für Korrespondenz:

patrick.steglich@th-wildau.de

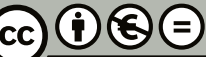

